\title{
Experimental Study, Characterization and Application of Starch-Graft- Acrylamide Gel for Plugging
}

\author{
Zhen LI ${ }^{1}$, Li CHENG ${ }^{1,2}{ }^{*}$, Ruiquan LIAO $^{1}$, Manlai ZHANG ${ }^{1}$, Kangwei ZHANG ${ }^{3}$, \\ Long YUAN ${ }^{3}$, Gang LIU $^{3}$
}

\author{
${ }^{1}$ The Branch of Key Laboratory of CNPC for Oil and Gas Production, College of Petroleum Engineering, Yangtze \\ University, Wuhan, China, 430100 \\ ${ }^{2}$ Lost Circulation Control Division, Oil and Gas Drilling Technology National Engineering Laboratory, Wuhan, China, \\ 430100 \\ ${ }^{3}$ Downhole Operation Company, PetroChina Dagang Oilfield Company, Tianjin, China, 300280
}

crossref http://dx.doi.org/10.5755/j01.ms.24.4.18565

Received 10 July 2017; accepted 04 January 2018

\begin{abstract}
During underbalance drilling, completion and workover wells, plugging channeling, blocking preformation and plugging formation water are inevitable problems. Gel is one of the most effective and convenient method to solve the problem. In this study, modified starch gel is synthesized, investigated experimentally and improved for efficient oil and gas field applications. The gel slurry is composed of starch $(3.6 \mathrm{wt} . \%)$, initiator $(0.02 \mathrm{wt} . \%)$, acrylamide (14.4 wt.\%), crosslinking agent (4.7 wt.\%), all of the components are mixed together with water at $\mathrm{pH} 10-11$ which viscosity is as low as $35-82 \mathrm{mPa}$.s and desired to form gel. Here the effects of the components, reaction temperature and $\mathrm{pH}$ on gelation time and gel viscosity are systematically investigated, and the results showed that the gelation can be controlled in a wide range $30-120 \mathrm{~min}$ efficiently by $\mathrm{pH}$ and initiator. Fourier Transform Infrared Spectroscopy (FTIR) and Scanning Electron Microscope (SEM) are employed to study the molecular structure and microstructure of the gel, respectively. A compact three-dimensional network structure was formed in the gel, which contribute to a good adhesion. The gel has been successfully used in shale gas field which provides a reference for sealing other similar high formation pressure under unbalanced workover treatment.
\end{abstract}

Keywords: modified starch gel, plug, snubbing service, microstructure.

\section{INTRODUCTION}

Underbalanced drilling and completion are becoming increasing increasingly utilized worldwide as an alternative technique to conventional overbalanced operation to reduce invasive near wellbores formation damage problems in oil and gas producing formations [1-5]. The underbalanced drilling technology can also have been implemented in the workover treatment if the situation such as water flow into the well and lost circulation are encountered. Water flows into the well is one of the main problems encountered while drilling top holes of onshore and offshores wells and when the water breaking though in the gas wells in the later period of gas field development. Lost circulation which occurs when hydrostatic pressure of fluid column in the wellbore is higher than the formation pressure is another common problem in the drilling operations and is usually accompanied by wellbore stability problems which can even result in the lost of well.

All of the problems mentioned above are usually attempted to be solved by mechanical shutoff or chemical treatment of selective intervals. Mechanical-set packer is usually used as a method for low damage completion of injection or disposal wells, and permanent mechanical zonal isolation for horizontal wells must be installed in the well at the completion stage using external casing packers or sliding side-doors, it is high priced and appears easily

\footnotetext{
* Corresponding author. Tel.: +86-18971635586; fax: +86-027-
} 69111050. E-mail address: chengli_whu.@163.com (L. Cheng) sealed and lax circumstance as well, especially horizontal wells and multilateral wells have long interval lengths and irregular shape, which means that they frequently suffer problems of uneven production from certain sections of the wells, due to wellbore pressure losses or changes in geological and reservoir parameters [6]. However, chemical treatment such gel can be applied remedially. First, gel can be easily pumped into any location of the well and satisfy various irregular shape liking a cork. Second, after temporary isolation by gel plug, it will not take much time to wash it out. Furthermore, mechanical packer installation cost much more time and money than gel.

For the last few decades, gel has been widely used in the petroleum and natural gas industry for different purposes including water shutoff, gas shutoff, casing repairs, conformance and profile control, permeability modification, leak resistance and sealing, plugging lost circulation zones, consolidation of loose formations [7-12]. Nevertheless, there are no reports of the use of modified starches as packer in underbalanced drilling and completion. In this study, a modified starch gel is designed and synthesized for plugging in snubbing service. Starch consists of two types of molecules amylose (normally 20-30\%) and amylopectin (normally 70-80\%). Both consist of polymers of $\alpha$-D-glucose unit in the ${ }^{4} C_{1}$ conformation. For the last few decades, the chemical modification of starch by graft copolymerization have been investigated widely and various works have been reported 
on the grafting of acrylamide [13], acrylonitrile [14], acrylic acid [15], methacrylates [16] onto starch [17]. However, no systematic study has been done for the gelation performance and microstructure. So in this study, environmentally friendly gel system with facile preparation is developed with new gel features of high viscosity and adjustable gelation time for snubbing service. In addition, a gas field test was also conducted to confirm that using modified starch gel is an effective plugged slug for pressure control. Though the laboratory experiments and gas field test, the gel will also further promote the application of snubbing service in mature oil field and gas field.

Hence, the present study emphasized on the facile preparation of modified starch gel and the process parameters such as proportion of raw material, $\mathrm{pH}$ and salt will be investigated and optimized. The slurry is liquid when prepared initially which viscosity is as low as $35-82 \mathrm{mPa}$.s, then formed a gel after a given temperature and a period of time. The gelation time and viscosity are studied to determine the best application for modified starch gel. The objective of this study was therefore to investigate the synthesis of modified starch gel with high viscosity and controllable gelatinization time, which could permit the potential use of gel made of this polymer in enhanced oil recovery.

\section{MATERIALS AND METHODS}

\subsection{Materials}

Corn starch (reagent-grade, Aladdin Industrial Corporation) was used for graft polymerization. Acrylamide (AR, Sinopharm Chemical) as monomer and ammonium persulfate as initiator (AR, Sinopharm Chemical) were used without further purification. Crosslinking agent is a compound consisting of amino groups, secondary amino group and triazine ring (a purity of $99 \%$, Aladdin Industrial Corporation) was used for cross-linking starch-g-AM. Sodium hydroxide (AR, Sinopharm Chemical) was used for adjusting $\mathrm{pH}$ value.

\subsection{Preparation of modified starch gel}

A definite amount of corn starch first dissolved in $80 \mathrm{~mL}$ of distilled water with stirring and heated within the temperature range of $80 \pm 2{ }^{\circ} \mathrm{C}$ for $30 \mathrm{~min}$ in the constant temperature water bath pot. Initiators, monomers (AM), crosslinking agent and curing agent were added into the reaction. After all the agents mixed well, adjusted $\mathrm{pH}$ value by slow-adding particles of $\mathrm{NaOH}$, then the reaction took place until completion to get a gel with high strength. The gelation time was recorded as the viscosity was increasing sharply.

\subsection{Measurements and analysis}

\subsubsection{Viscosity measurements}

AR2000ex rheometer (TA Instruments Ltd., Crawley, UK) with aluminum parallel plate geometry (40 mm diameter, $1 \mathrm{~mm}$ gap) was used to carry out the rheological measurements. The AR2000ex is a stress controlled rheometer equipped with a force rebalance transducer. Viscous properties of samples were measured through strain sweep tests carried out at $1 \mathrm{~Hz}$. The temperature of the samples was controlled by using a water bath connected to the bottom plate. The temperature of the water was controlled by a Peltier system. Each sample was allowed to equilibrate for $2 \mathrm{~min}$ before the test carried out.

\subsubsection{Fourier Transform Infrared Spectroscopy (FTIR)}

The reaction product was washed with distilled water several times to remove any ungrafted molecules, monomer and base until the solution $\mathrm{pH}=7$. The washed product was dehydrated and precipitated with methanol and dried at $65^{\circ} \mathrm{C}$ until the weight of the specimen was constant. Then the solid was crushed into fine powder. Fourier Transform Infrared Spectroscopy of the proceeded products using a $\mathrm{KBr}$ pellet were recorded on Avatar E.S.P. spectrometers, Nicolet Ltd, USA in the range of $4000-400 \mathrm{~cm}^{-1}$.

\subsubsection{Scanning electron microscopy (SEM)}

The surface morphology and topography of corn starch and crosslinked starch-g-AM were evaluated by scanning electron microscope (JSM-6390lv, Jeol, Japan). Before examination, the samples were mounted onto stubs using double-sided dried adhesive carbon tape and vacuum coated with gold palladium film (thickness $2 \mathrm{~nm}$ ) by sputter coater (Edward S-150, UK) to make them electrically conductive. Representative sections were photographed for evaluation.

\section{RESULTS AND DISCUSSION}

\subsection{Effect of the initiator on the gel performance}

Considering the process conditions and the cost of huge amount of applications in oil and gas field, ammonium persulfate (APS) was chosen as the initiator. In this experiment, the gel solution was composed of starch (3.6 wt.\%), acrylamide (10.8 wt.\%), cross-linking agent (4.7 wt.\%), while initiator concentrations were varied from $0.002 \%$ to $0.5 \%$, and the gel system was initiated at $80{ }^{\circ} \mathrm{C}$. During the reaction APS could produce hydrogen sulfate free radical, while free radicals on the starch are not formed initially, they are produced through the chain transfer reaction with an existing free radical, meanwhile significant amounts of homopolymer and free copolymer are also formed by the reaction of initially formed radicals with monomer(s) before chain transfer to starch could occur [18]. Fig. 1 shows the effect of the initiator on gel properties.

It can be seen from Fig. 1 that, as the concentration of APS increased, the viscosity of gel increased first and then decreased. It benefited from the growth of the chain length, the acceleration of the polymerization reaction and the combination reaction between them will rise, so as the viscosity increasing. But redundant initiator concentration can lead to excessive free radicals. Based on steady-state theory and free radical polymerization reaction kinetics, when the trigger rate is too high, both the termination rate of the grafting reaction and the percentage of free polymers with short chain length will increase with increasing concentration of the initiator due to the fixed amount of 
monomer, which will result in the decreasing of viscosity and the decline of gelation time. The maximum of gel viscosity was about $2 \times 10^{6} \mathrm{mPa}$.s. According to the practice application, the reaction time was controlled for at least $30 \mathrm{~min}$ at $80{ }^{\circ} \mathrm{C}$ and for the viscosity, the higher the better, so in the present work, the concentration of APS was selected at $0.02 \mathrm{wt} . \%$.

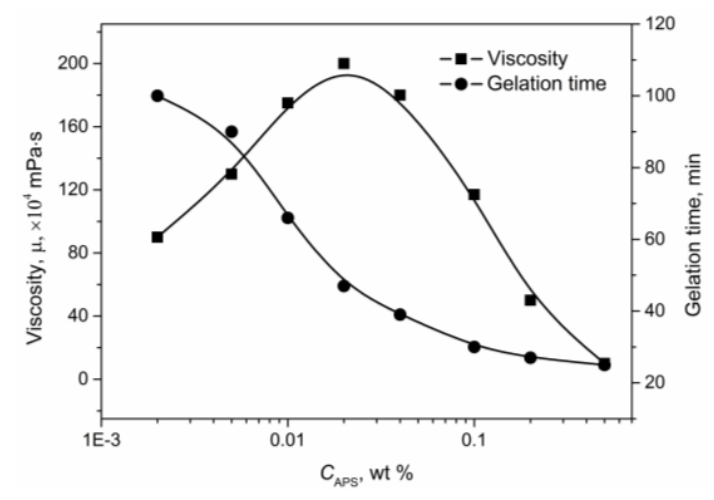

Fig. 1. The influence of the concentration of initiator

\subsection{Effect of monomer-to-starch ratio on the gel performance}

$\mathrm{AM}$ is selected for copolymerization monomer since it easily forms hydrogen-bonds with more water to be expected to obtain the copolymer with greater water absorption capacity so as to decrease syneresis and make sure stable performance at the condition of high temperature and high formation pressure. Different amount of monomer AM was added in the graft polymerization procession at $80{ }^{\circ} \mathrm{C}$ while starch (3.6 wt.\%), initiator (0.02 wt. \%) and cross-linking agent (4.7 wt.\%) were added in the gel solution. The effect of AM-to-starch ratio on the performance of gel is presented in Fig. 2.

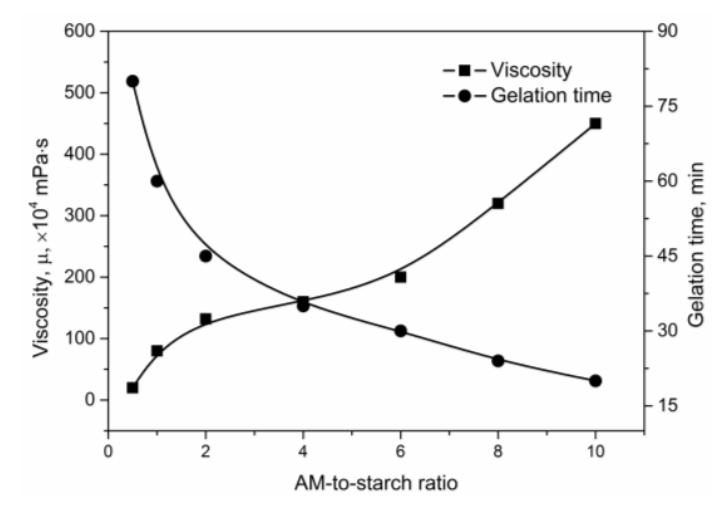

Fig. 2. Effect of AM-to-starch ratio on the gel performance

As can be seen from the Fig. 2., the gelation time was shortened and the viscosity of the gel increased as AM-to-starch ratio increased. As at a lower amount of AM, a small number of monomer can diffuse to graft onto the starch backbone, and the increasing of the monomer concentration was beneficial to graft polymer chain. On one hand, it was advantageous for monomers to spread to the active site of the long chain of starch, forming the graft copolymer and improving the efficiency of grafting. On the other hand, the graft copolymerization with a higher grafting efficiency gives the graft copolymer containing higher amount of hydrophilic chains with large length.
Thus the viscosity increased due to the increasing amount of intramolecular and intermolecular hydrogen bonds. In addition, the viscosity also increased because the free water content is reduced [19].

Because hydroxyl group on the starch chain was used to graft copolymerization with AM, a proportion of AM to corn starch should not to too low. Moreover, considering the gelation time, viscosity and cost, the optimum ratio of AM to corn starch was controlled at 4:1.

\subsection{Effect of $\mathrm{pH}$ on gel performance}

In consideration of the acid-base properties of on-site water collection in the oilfield or gasfield, the effect of $\mathrm{pH}$ on gel was investigated here. The influence of selected $\mathrm{pH} 7-13$ on the gel performance (starch (3.6 wt.\%), initiator (0.02 wt.\%), acrylamide (14.4 wt.\%), crosslinking agent $\left(4.7\right.$ wt. \%), at $80^{\circ} \mathrm{C}$ ) is shown in Fig. 3 .

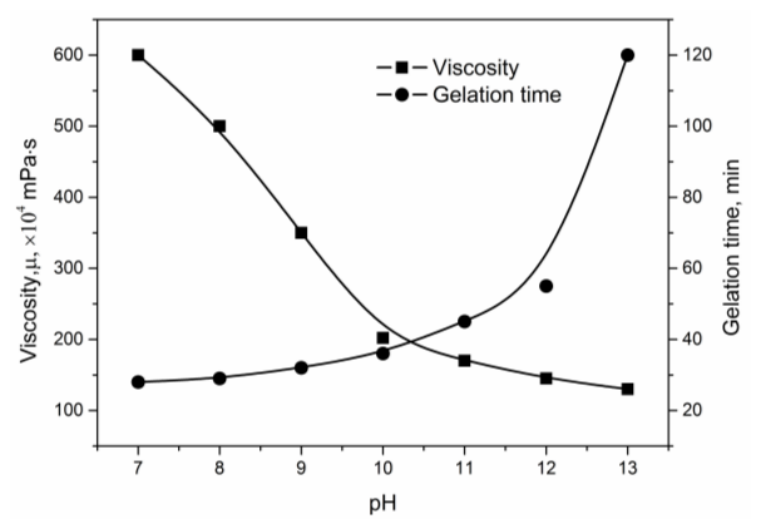

Fig. 3. Effect of $\mathrm{pH}$ on the gel performance

When the system was under alkaline condition, the viscosity of system decreased with the increasing of $\mathrm{pH}$ value while gelation time increases. With the increase of the amount of $\mathrm{NaOH}$, large amount of $-\mathrm{CONH}_{2}$ group are converted into $-\mathrm{COOH}$ or $-\mathrm{COONa}$ groups due to the hydrolysis reaction according to the Eq. 1.

$$
\mathrm{R}-\mathrm{CONH}_{2}+\mathrm{NaOH} \rightarrow \mathrm{R}-\mathrm{COONa}+\mathrm{NH}_{3} .
$$

R-COONa can be considered as a weak alkali, and there is a hydrolysis equilibrium shown as Eq. 2 [18, 20].

$$
\mathrm{R}-\mathrm{COONa}+\mathrm{H}_{2} \mathrm{O} \leftrightarrow \mathrm{R}-\mathrm{COOH}+\mathrm{NaOH} \text {. }
$$

The decrease of the amount of $-\mathrm{CONH}_{2}$ could reduce the opportunity of reaction between -OH groups on starch and $-\mathrm{CONH}_{2}$ group on acrylamide chain to take place, which lead in the extension of gelation time. At higher $\mathrm{pH}$ values, the concentration of anionic groups in the polymer network increases according to Eq. 1 and Eq. 2, so the electrostatic repulsion between the graft copolymer becomes stronger which will lower the crosslink density of gel, as a result the viscosity decreases and the gelation time was extended. Fortunately, it was obvious that the gelation time and viscosity can be controlled by $\mathrm{pH}$ adjusting, which give the gel a broader range of application. As a result, the optimal $\mathrm{pH}$ was selected $10-11$.

\subsection{Effect of reaction time on the viscosity}

The onset time of gelation is an important parameter in understanding reaction mechanism in the network- 
formation system, because in the practical application it should leave enough time before the reaction takes place, that is make sure all the reactants will be pumped to the destination before gelatinization. In the published literatures, some determined the gelation time as the time that gel materials could be detected by solvent extraction [21-24], others defined it as the moment that the reaction solution lost its mobility in a tube at an upside-down position for $10 \mathrm{~s}$ [25-28]. In this work, the gelation time was determined by the sharp variation of complex viscosity of the reactant fluent during the polymerization. The relation between time and viscosity is studied shown in Fig. 4.

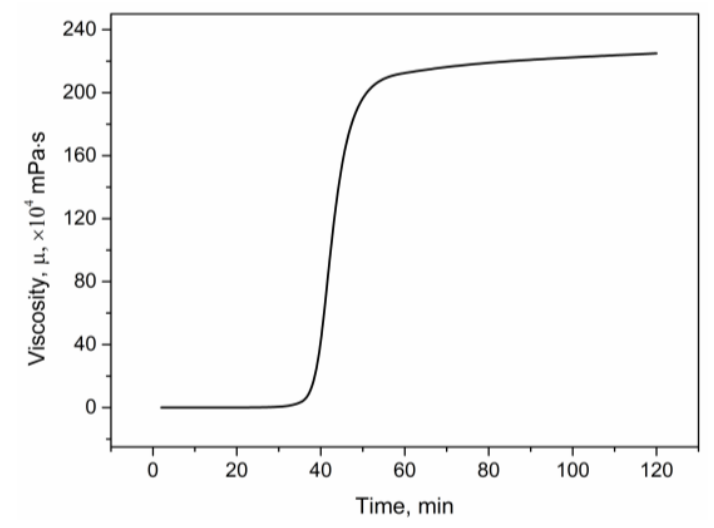

Fig. 4. Variation of viscosity during gelatinization

The curve depicted in Fig. 4 shows that under the conditions applied, the value of viscosity was very small about $40 \pm 5 \mathrm{mPa}$.s and had remained constant for $38 \mathrm{~min}$ at the beginning of reaction, followed by an abrupt acceleration upon the network formation, after that the growth became gradually again for a long time. This phenomenon can be explained by slow initiation, fast propagation, fast termination and fast crosslinking [22-24]. In the reaction, corn starch and monomer AM were initiated slowly within a period of time at the early stage of grafting and polymerization. Then these short chains grew rapidly through propagation with vinyl groups in monomers. At a higher conversion, these chains combine one another though crosslinking to form huge networks resulting in an abrupt increase in viscosity. It is when the gel is formed that the viscosity will maintain a steady standard.

The viscosity is related to how easy the solution of reactants will be pumped to the destination in the predetermined length of time. In the field application, the smaller the primary viscosity is, the better it is. Hence, it is desired not bigger than $300 \mathrm{mPa} . \mathrm{s}$, because under high pressure the fluid with high viscosity is difficult to pump into the wellbore. Under the reaction condition shown in Fig. 4., the pumping process should have been finished within 38 min to avoid the gelatinization taking place. The optimum gelation time at different temperature could be obtained through the adjustment of $\mathrm{pH}$ as well as the amount of initiator.

\subsection{Effect of salt on gel performance}

In the consideration of application condition, the evaluation of effect of salt, especially divalent metal ions, on gel performance was carried out. The gel solution was prepared with high concentration of $\mathrm{Mg}^{2+}$ and $\mathrm{Ca}^{2+}$, as high as $10000 \mathrm{ppm}$, respectively, and the viscosities were shown in Fig. 5.

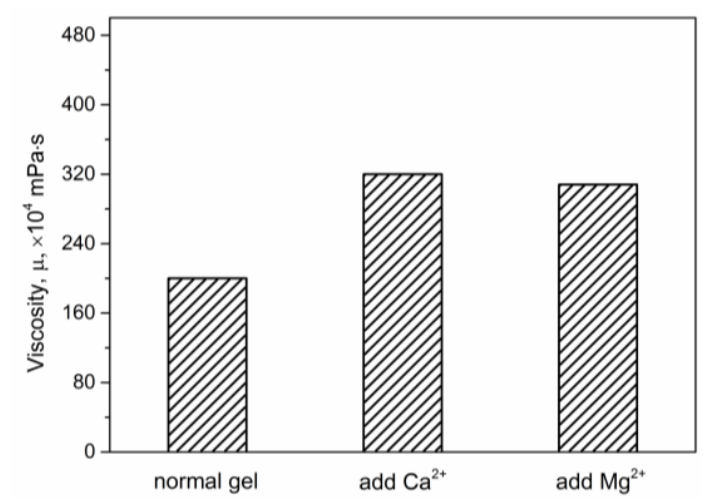

Fig. 5. Effect of $\mathrm{Ca}^{2+}$ and $\mathrm{Mg}^{2+}$ on viscosity of gel

Compared with the empty sample, the viscosity of gel increases with addition of ions in the solution. This phenomenon can be explained from two aspects. Firstly, the presence of ions in the solution could counteracts the mutual repulsion of the original ion in the network itself though the intramolecular and intermolecular complex formations ability of carboxamide and carboxylate groups $[29,30]$. Secondly, the divalent ion is able to neutralize the charges which could weaken the electrostatic repulsion between charged groups inside the gel. Consequently, the excellent gelling properties are obtained under the condition of high salinity, which can be due to enhancement of the crosslink density of the network.

\subsection{FT-IR spectra of the polymers}

The infrared spectra of the compounds under investigation were recorded in the $4000-500 \mathrm{~cm}^{-1}$ region with $\mathrm{KBr}$ pellets. The functional groups of starch and crosslinked St- $g$-AM were characterized by FTIR as shown in Fig. 6.

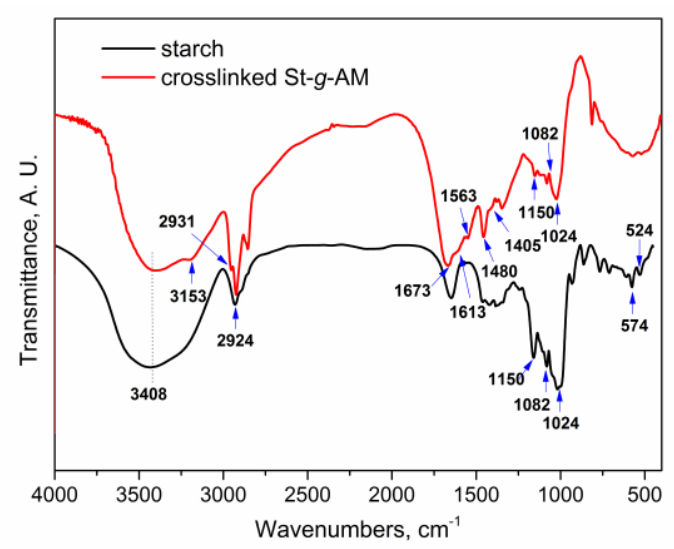

Fig. 6. FTIR spectra of corn starch and crosslinked St-g-AM

FTIR spectra of starch and crosslinked St-g-AM give all the characteristic absorption bands of corn starch $\left(3408 \mathrm{~cm}^{-1}\right.$ (s, broad); $\left.2924 \mathrm{~cm}^{-1}(\mathrm{~m})\right) ; 1150,1082$ and $1024 \mathrm{~cm}^{-1}$ (s) for $\mathrm{O}-\mathrm{H}, \mathrm{C}-\mathrm{H}$ and $\mathrm{C}-\mathrm{O}$ stretching, respectively) [19,31]. After the reaction the absorption peak at 574 and $524 \mathrm{~cm}^{-1}$ attributed to $-\mathrm{OH}$ group on starch 
become smaller, this indicated that the $\mathrm{OH}$ groups on starch are converted during the reaction. The absorption bands at 3153,1673 and $1613 \mathrm{~cm}^{-1}$ attributed to the $\mathrm{CONH}_{2}$ group on the acrylamide also change during the reaction and a absorption peak at $1405 \mathrm{~cm}^{-1}$ attributed to $\mathrm{CO}_{2}$ group appeared [18]. The IR spectrum of acrylamide grafted starch showed distinctive and characteristic absorption bands around $\left(3500-3545 \mathrm{~cm}^{-1}\right)$ for $v\left(\mathrm{NH}_{2}\right)$ and $\left(2931 \mathrm{~cm}^{-1}\right)$ for stretching band for methylene group $[32,33]$. All these bands mentioned above indicated the presence of the polyacrylamide chains grafted on starch.

As shown in Fig. 6, the distinct bands at 1563 and $1480 \mathrm{~cm}^{-1}(\mathrm{C}=\mathrm{N}$ vibrations of the s-triazine ring $)$ and there is an absorption indicative of a secondary amine at $1588 \mathrm{~cm}^{-1}$ in the fingerprint region confirm the successful incorporation of triazine units provided by the crosslinker into the network [34, 35].

\subsection{Surface morphology of the gel}

The surface morphology, structure of the starch and the representative crosslinked st- $g$-AM were studied by scanning electron microscopy (SEM) as shown in Fig. 7.
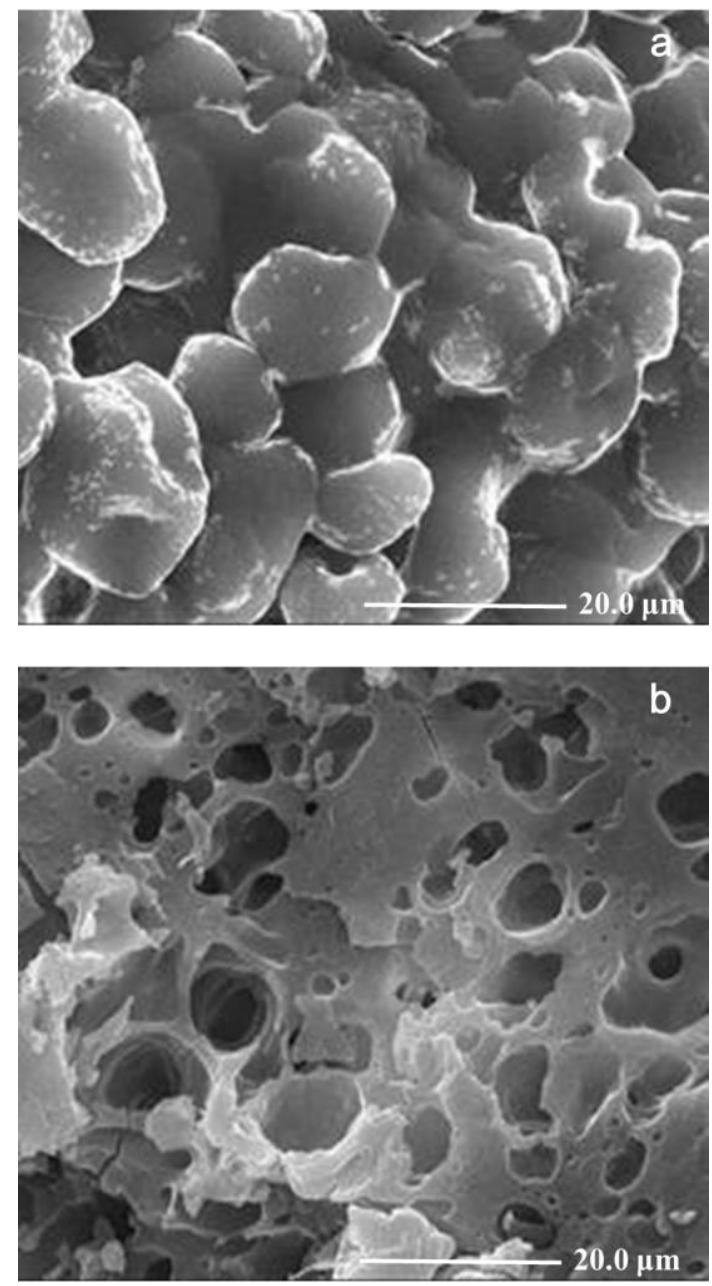

Fig. 7. SEM photographs: $\mathrm{a}-$ corn starch; $\mathrm{b}-$ crosslinked starchg-AM: $80^{\circ} \mathrm{C}, \mathrm{pH} 10$, AM-to-starch ratio 4, APS $0.02 \%$

Fig. 7 a shows that the corn starch particles have a smooth and approximately spherical surface before grafting and crosslinking reaction, while after the reaction almost all of the starch particles were disrupted and formed many cavities inside the gel shown in Fig. $7 \mathrm{~b}$. This phenomenon can also be explained through FTIR spectra of starch and crosslinked st- $g$-AM. The change of the characteristic peaks of -C-O-C- and nearly disappearance of $-\mathrm{OH}$ groups absorption peak could prove the degradation of starch particles; as a result, the spheres was not found in Fig. 7 b. Besides, the porosity can be explained by Eq. 1 that the hydrolysis reaction of amide groups that produced $\mathrm{NH}_{3}$ under the alkaline condition, this gaseous product diffusion out of the product favor the formation of pores. This porosity is expected to enhance adhesive property of the crosslinked starch-g-AM gel, since, as is well known, the topography, the surface charge and interconnected pores are insuring good adhesion [36]. Fig. $7 \mathrm{~b}$ also shows the crosslinked polymer have a continuous and dense 3D structure which can contribute to the high elasticity of the gel. Fig. 8 is a schematic illustration to show the network.

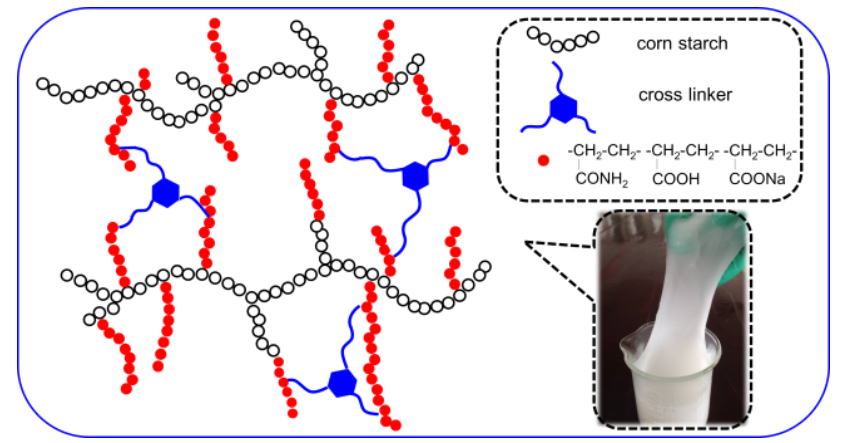

Fig. 8. Schematic illustration of the crosslinked starch-g-AM and the modified starch gel for the inserted picture

\subsection{Field application}

The gel as a packer was successfully applied in the shale gas field of China. The horizontal well named Longye $1 \mathrm{HF}$ as a production well has operated for nearly 10 years and was found that the phenomenon of bottom water coning was very serious in April 2017 as shown in Fig. 9, so the coiled tubing had to be pull out from the well to resume production through casing.

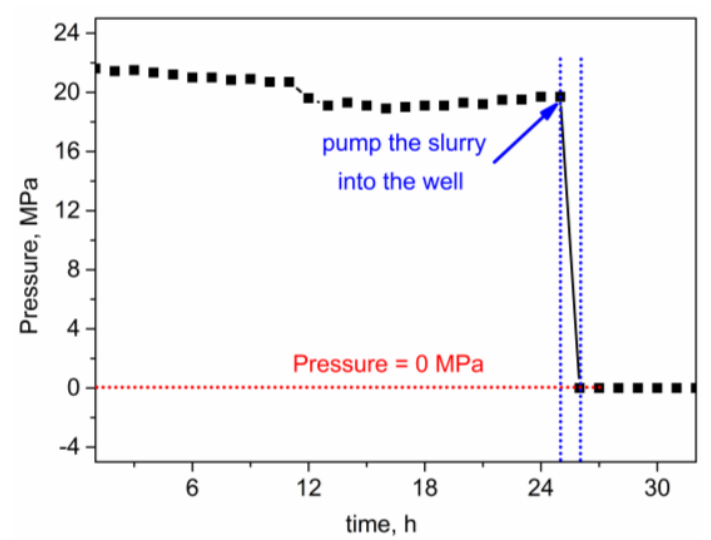

Fig. 9. The wellhead pressure curve of Longye $1 \mathrm{HF}$

During the treatment process, about $1 \mathrm{~m}^{3}$ ahead fluid and $0.5 \mathrm{~m}^{3}$ of gel solution were injected into coiled tubing at a depth of about of $3500 \mathrm{~m}$ and $3000 \mathrm{~m}$, and then the well was shut off for about $1 \mathrm{~h}$ waiting for the gel to be fully formed. After this, the tubing pressure directly 
changed to zero (Fig. 9) and the tubbing was safely and successfully pulled out from the well. The wellhead pressure of zero was continuously observed for 3 days which indicated the gel presents a stable and reliable performance. The result proves that the gel plug as a packer slug was an effective treatment for snubbing service.

\section{CONCLUSIONS}

The novel gel with high viscosity composed by crosslinked starch- $g$-AM was synthesized by modifying grafted copolymerization of corn starch and AM, and the preparation conditions and synthesis techniques were discussed. The conclusions presented are as follows.

1. The dominant factor that effects the gel viscosity is the concentration of monomers, so dosage of AM could be changed to meet the needs of filed application.

2. The main factor that effects the gelling time is $\mathrm{pH}$ value, which could be adjusted to control time and suit the field operation need, the adjusted gelation time ensures that the gel solution enters the destination before becoming an unmovable plug, moreover the high gel viscosity gives the gel enough strength to plug high pressure. Above all, the optimal $\mathrm{pH}$ was selected $10-11$.

3. The slurry is liquid when prepared initially which viscosity is as low as $35-82 \mathrm{mPa}$.s and the formula of gel is simple, various components and $\mathrm{pH}$ value could be confirmed easily before pumping into the wellbore, then formed a gel at a given temperature and a period of time.

4. Salt addition can compact the density of the network structure through screening of the charges, causing a significant lift in viscosity and confirming the involvement of electrostatic interactions.

5. The morphology of gel was observed by SEM, and it was found that the porosity and dense continuous phase could contribute to the high viscosity and elasticity, respectively.

These gels represent an important step towards the design and application of physical gels for oilfield and gasfield, since initiator, $\mathrm{pH}$ and ionic strength sensitivity of structure could be used to insure secure application conditions. Moreover, they are of interest as gelling agents with high viscosity and time controllable property which opens an important door to the study of this kind of environmental and easily pumped gels aimed to be used as smart plug for well completion engineering and in other applications in the field of oil and gas exploitation.

\section{Acknowledgments}

The authors thank all the financial support. This work was supported by the National Natural Science Foundation of China (NSFC NO. 51404039) and PetroChina Innovation Foundation (NO. 2014D-5006-0309). Valuable comments and suggestions from the reviewer are also gratefully acknowledged.

\section{REFERENCES}

1. Hu, Y., Guo, W.L. Underbalanced Drilling Basic Theory and Practice. Petroleum Industry Press, Beijing, 2009.

2. Aarsnes, U.J.F., Meglio, F.D., Aamo, O.M., Kaasa, G.O. Fit-for-Purpose Modeling for Automation of Underbalanced Drilling Operations Managed Pressure Drilling \& Underbalanced Operations Conference \& Exhibition 2014. https://doi.org/10.2118/168955-MS

3. Nee, L.S., Khalil, M., Jan, B.M., Ali, B.S. Lightweight Biopolymer Drilling Fluid for Underbalanced Drilling: An Optimization Study Journal of Petroleum Science \& Engineering 129 2015: pp. 178-188. https://doi.org/10.1016/j.petrol.2015.03.006

4. Pedersen, T., Godhavn, J.M., Schubert, J. Supervisory Control for Underbalanced Drilling Operations IFACPapersOnLine 48-6 2015: pp. 120-127. https://doi.org/10.1016/j.ifacol.2015.08.019

5. Garrouch, A.A., Lababidi, H.M.S. Development of an Expert System for Underbalanced Drilling Using Fuzzy Logic Journal of Petroleum Science \& Engineering 31 (1) 2001: pp. $23-39$. https://doi.org/10.1016/S0920-4105(01)00136-X

6. Jones, A.T., van der Bas, F., Hardy, M. Gel Plugs for Temporary Isolation in Horizontal Wells Completed with Slotted Liners. In: Presented at the 1997 SPE European Formation Damage Conference held in Hague (SPE 38199), Netherlands, 2 - 3 June, 1997. https://doi.org/10.2118/38199-MS

7. Sharifpour, E., Escrochi, M., Riazi, M., Ayatollahi, S. On the Importance of Gel Rigidity and Coverage in A Smart Water Shutoff Treatment in Gas Wells Journal of Natural Gas Science \&Engineering 31 2016: pp. 808-818. https://doi.org/10.1016/j.jngse.2016.03.001

8. Karimi, S., Esmaeilzadeh, F., Mowla, D. Identification and Selection of a Stable Gel Polymer to Control or Reduce Water Production in Gas Condensate Fields Journal of Natural Gas Science \&Engineering 21 2014: pp. $940-950$ https://doi.org/10.1016/j.jngse.2014.10.026

9. Saavedra, N.F., Mamora, D.D., Burnett, D.B., Platt, F.M. Chemical Wellbore Plug for Zone Isolation in Horizontal Wells. In: Presented at the 1998 SPE/DOE Improved Oil Recovery Symposium held in Tulsa (SPE 39647), Oklahoma, 19-22 April, 1998. https://doi.org/10.2118/39647-MS

10. Xiang, X.W., Zhai, C., Xu, Y.M., Yu, X., Xu, J.Z. A Flexible Gel Sealing Material and A Novel Active Sealing Method for Coal-bed Methane Drainage Boreholes Journal of Natural Gas Science \&Engineering 26 2015: pp. 1187-1199. https://doi.org/10.1016/j.jngse.2015.08.016

11. Ay, A., Gucuyener, I.H., Kök, M.V. An Experimental Study of Silicate-polymer Gel Systems to Seal Shallow Water Flow and Lost Circulation Zones in Top Hole Drilling Journal of Petroleum Science \& Engineering 122 (3) 2014: pp. 690-699. https://doi.org/10.1016/j.petrol.2014.09.011

12. Burns, D., Burns, M., Wilhite, P.M., Cool, S., Lawrence, K.S., Oglesby, K., Glass, J. New Generation Silicate Gel System for Casing Repairs and Water Shutoff, paper SPE 113490. SPE/DOE Improved Oil Recovery Symposium, Tulsa, Oklahoma. April 19-23, 2008. https://doi.org/10.2118/113490-MS 
13. Cheng, L., Chen, K.K., Zhang, M.L., Liu, D.J., Liao, R.Q., Gu, Y. Oxidized/crosslinking Double-Modified Starch Gel Oil Drilling \& Production Technology 37 (3) 2015: pp. 106-109.

14. Chauhan, G.S., Jaswal, S.C., Verma, M. Post Functionalization of Carboxymethylated Starch and Acrylonitrile Based Networks Through Amidoximation for Use as Ion Sorbents Carbohydrate Polymer 66 (4) 2006: pp. $435-443$. https://doi.org/10.1016/j.carbpol.2006.03.030

15. Kolya, H., Tripathy, T. Hydroxyethyl Starch-g-Poly-(N,Ndimethylacrylamide-co-acrylic acid): An Efficient Dye Removing Agent European Polymer Journal 49 (12) 2013: pp. $4265-4275$. https://doi.org/10.1016/j.eurpolymj.2013.10.012

16. Li, M.C., Ge, X., Cho, U.R. Mechanical Performance, Water Absorption Behavior and Biodegradability of Poly(methyl methacrylate)-Modified Starch/SBR Biocomposites Macromolecular Research 21 (7) 2013: pp. $793-800$. https://doi.org/10.1007/s13233-013-1088-4

17. BeMiller, J.N. Pasting, Paste, and Gel Properties of StarchHydrocolloid Combinations Carbohydrate Polymers 86 (2) 2011: pp. 386-423. https://doi.org/10.1016/j.carbpol.2011.05.064

18. Wu, J.H., Wei, Y.L., Lin, J.M., Lin, S.B. Study on StarchGraft-Acrylamide/mineral Powder Superabsorbent Composite Polymer 44 (21) 2003: pp. 6513-6520. https://doi.org/10.1016/S0032-3861(03)00728-6

19. Lanthong, P., Nuisin, R., Kiatkamjornwong, S. Graft Copolymerization, Characterization, and Degradation of Cassava Starch-g-Acrylamide/itaconic Acid Superabsorbents Carbohydrate Polymer 66 (2) 2006: pp. 229-245. https://doi.org/10.1016/j.carbpol.2006.03.006

20. Tong, Z., Liu, X.X. Swelling Equilibria and Volume Phase Transition of Partially Neutralized Poly(acrylic acid) Gels European Polymer Journal 29 (5) 1993: pp. 705 -709. https://doi.org/10.1016/0014-3057(93)90131-X

21. Jiang, C.F., Shen, Y.Q., Zhu, S., Hunkeler, D. Gel Formation in Atom Transfer Radical Polymerization of 2(N,N-dimethylamino)ethyl Methacrylate and Ethylene Glycol Dimethacrylate Journal of Polymer Science Part A Polymer Chemistry 39 (21) 2001: pp. 3780-3788. https://doi.org/10.1002/pola.10023

22. Yu, Q., Zhang, J.Z., Cheng, M.L., Zhu, S.P. Kinetic Behavior of Atom Transfer Radical Polymerization of Dimethacrylates Macromolecular Chemistry \& Physics 207 (3) 2006: pp. 287-294. https://doi.org/10.1002/macp.200500378

23. Yu, Q., Qin, Z.Q., Li, J.C., Zhu, S.P. Diffusion-Controlled Atom Transfer Radical Polymerization with Crosslinking Polymer Engineering \& Science 48 (7) 2008: pp. $1254-1260$. https://doi.org/10.1002/pen.21082

24. Yu, Q., Xu, S.J., Zhang, H.W., Ding, Y.H., Zhu, S.P. Comparison of Reaction Kinetics and Gelation Behaviors in Atom Transfer, Reversible Addition-Fragmentation Chain Transfer and Conventional Free Radical Copolymerization of Oligo(ethylene glycol) Methyl Ether Methacrylate and Oligo(ethylene glycol) Dimethacrylate Polymer $50(15)$ 2009: pp. 3488-3493.

https://doi.org/10.1016/j.polymer.2009.05.032
25. Ide, N., Fukuda, T. Nitroxide-Controlled Free-Radical Copolymerization of Vinyl and Divinyl Monomers. 2. Gelation Macromolecules 32 (1) 1999: pp. 95-99. https://doi.org/10.1021/ma9805349

26. Gao, H.F., Min, K., Matyjaszewski, K. Determination of Gel Point during Atom Transfer Radical Copolymerization with Cross-Linker Macromolecules 40 (22) 2007: pp. $7763-7770$. https://doi.org/10.1021/ma071324b

27. Gao, H.F., Li, W.W., Matyjaszewski, K. Synthesis of Polyacrylate Networks by ATRP: Parameters Influencing Experimental Gel Points Macromolecules 41 (7) 2008: pp. $2335-2340$. https://doi.org/10.1021/ma702823b

28. Brannister, I., Billingham, N.C., Armes, S.P., Rannard, S.P., Findlay, P. Development of Branching in Living Radical Copolymerization of Vinyl and Divinyl Monomers Macromolecules 39 (22) 2006: pp. 7483-7492. https://doi.org/10.1021/ma061811b

29. Castel, D., Ricarb, A., Audebert, R. Swelling of Anionic and Cationic Starch-based Superabsorbents in Water and Saline Solutions Journal of Applied Polymer Science 39 (1) 1990: pp. $11-29$. https://doi.org/10.1002/app.1990.070390102

30. Caykara, T., Bozkaya, U., Kantoglu, O. Network Structure and Swelling Behavior of Poly(acrylamide/crotonic acid) Hydrogels in Aqueous Salt Solutions Journal of Polymer Science Part B Polymer Physics 41 (14) 2003: pp. $1656-1664$. https://doi.org/10.1002/polb.10500

31. Song, W.Q., Guo, Z.C., Zhang, L.Q., Zheng, H.J., Zhao, Z.W. Antibacterial Activity of Starch/acrylamide/allyl Triphenyl Phosphonium Bromide Copolymers Synthesized by Gamma Irradiation Radiation Physics \& Chemistry 91 (10) 2013: pp. 114-119. https://doi.org/10.1016/j.radphyschem.2013.06.003

32. Silverstein, R., Webster, F., Kiemle, D. Spectrophotometric Identification of Organic Compounds (7th ed.). New York: John Willy and Sons, 2005.

33. Al-Karawi, A.J.M., Al-Daraji, A.H.R. Preparation and Using of Acrylamide Grafted Starch as Polymer Drug Carrier Carbohydrate Polymer 79 (3) 2010: pp. $769-774$ https://doi.org/10.1016/j.carbpol.2009.10.003

34. Yang, G.W., Han, H.Y., Du, C.Y., Luo, Z.H., Wang, Y.J. Facile Synthesis of Melamine-based Porous Polymer Networks and Their Application for Removal of Aqueous Mercury Ions Polymer 51 (26) 2010: pp. 6193-6202. https://doi.org/10.1016/j.polymer.2010.10.052

35. Lavric, S., Kocar, D., Mihelic, I., Braybrook, C. Accurate Mass Determination of Melamine-Formaldehyde Synthetic Polymers after Separation on Preparative HPLC Progress in Organic Coatings 81 2015: pp. 27-34. https://doi.org/10.1016/j.porgcoat.2014.12.010

36. Vieira, J.N., Posada, J.J., Rezende, R.A., Sabino, M.A. Starch and Chitosan Oligosaccharides as Interpenetrating Phases in Poly(N-isopropylacrylamide) Injectable Gels Materials Science and Engineering $C \quad 37$ (1) 2014: pp. 20-27. https://doi.org/10.1016/j.msec.2013.12.005 